\title{
Identification of the Commercially Important Oreosomatid Fish (Zeiformes: Teleostei) of the Emperor Seamounts, with Comments on Diagnostic Characters of the Species
}

\author{
Koichi Hoshino ${ }^{1,6}$, Kunpei Kosaka ${ }^{2}$, Kota Sawada ${ }^{3,4}$, and Masashi Kiyota ${ }^{3,5}$ \\ ${ }^{1}$ Fisheries Technology Institute, Japan Fisheries Research and Education Agency, 1551-8 Taira-machi, Nagasaki 851-2213, Japan \\ E-mail: hoshinok@affrc.go.jp \\ ${ }^{2}$ Tokyo University of Marine Science and Technology, 4-5-7 Kohnan, Minato-ku, Tokyo 108-8477, Japan \\ ${ }^{3}$ National Research Institute of Far Seas Fisheries, Japan Fisheries Research and Education Agency, \\ 2-12-4 Fukuura, Kanazawa-ku, Yokohama, Kanagawa 236-8648, Japan \\ ${ }^{4}$ Present Address: Fisheries Resources Institute, Japan Fisheries Research and Education Agency, \\ 2-12-4 Fukuura, Kanazawa-ku, Yokohama, Kanagawa 236-8648, Japan \\ ${ }^{5}$ Present Address: Graduate School of Fisheries and Environmental Sciences, Nagasaki University, \\ 1-14 Bunkyo-machi, Nagasaki 852-8521, Japan \\ ${ }^{6}$ Corresponding author
}

(Received 16 June 2021; Accepted 31 August 2021)

\begin{abstract}
An oreosomatid of the genus Allocyttus McCulloch, 1914 is fished commercially on the Emperor Seamounts. However, the species' identity is uncertain, as is the taxonomy of the oreosomatid species of the seas around Japan, where the names Allocyttus verrucosus Gilchrist, 1906 (type locality: off South Africa) and A. folletti Myers, 1960 (type locality: off California) have both been used. From its anticipated susceptibility to over-exploitation, it is urgent to establish the correct taxonomic identity to facilitate effective management measures. Meristics, morphometrics and scale characters of the specimens from the Emperor Seamounts and Japan agreed well with data of the holotype of $A$. folletti and differed from those of $A$. verrucosus, confirming that those specimens represent $A$. folletti. Oreosomatids reported from the western North $\mathrm{Pa}$ cific in the literature were identified as $A$. folletti. From the data of the present study and historical references, $A$. folletti is thought to be distinguished from $A$. verrucosus by the following characters: more dorsal- and anal- spines + rays (36-42 vs. 33-38 and 31-35 vs. 27-33 respectively), more total vertebrae (37-41 vs. 34-38), greater numbers of enlarged scales of dorsal- (S-DFB) and anal-fin base (S-AFB) (31-42 vs. 26-31, and 29-37 vs. 25-28 respectively), more spines on the margin of S-DFB and S-AFB (up to 7-12 vs. 3-6), a shorter preanal-fin length (53.8-63.6\% vs. 64.8-83.7\% of SL), a longer caudal peduncle (10.4-15.6\% vs. $6.1-10.2 \%$ of SL), a shorter head (32.9-40.4\% vs. $38.5-48.4 \%$ of SL), and cycloid scales on the mid-side of body (vs. ctenoid). Available data indicate that A. folletti reaches up to $537 \mathrm{~mm}$ SL, larger than A. verrucosus (up to ca. $325 \mathrm{~mm} \mathrm{SL}$ ). From the anticipated slow growth and longevity, concern is raised regarding the susceptibility of $A$. folletti to over-exploitation.
\end{abstract}

Key Words: Allocyttus folletti, Allocyttus verrucosus, Oreosomatidae, northwestern Pacific.

\section{Introduction}

Members of the genus Allocyttus McCulloch, 1914 of the family Oreosomatidae (Zeiformes), commonly known as "oreos," are deep-sea benthopelagic (Karrer 1986a, b; Heemstra 2016) fishes widely distributed in the waters of seamounts and continental slopes of the Pacific, Atlantic and Indian Oceans (James et al. 1988). The monophyly of the Oreosomatidae is demonstrated by the phylogenetic studies (Tyler et al. 2003; Tyler and Santini 2005; Grande et al. 2018). Within the family, Allocyttus is characterized by having a deep and compressed body, a large eye (but its diameter $52 \%$ or less of head length), the first dorsal-fin spine shorter than the second, and the predorsal profile nearly straight without an abrupt rise anterior to the dorsal fin (James et al. 1988). The juveniles are pelagic, and significantly morphologically distinct from the adults in having a swollen abdomen with two rows of cone-like scutes, which are reduced but may remain in adults as patches of enlarged scales between the pectoral and pelvic fins (Kobayashi et al. 1968; Hart 1973; James et al. 1988).

Four species are recognized in the genus: Allocyttus verrucosus (Gilchrist, 1906), A. folletti Myers, 1960, A. guineensis Trunov and Kukuev, 1982, and A. niger James, Inada, and Nakamura, 1988. Allocyttus niger is distributed in the South West Atlantic and Southern Indian Oceans, and off Southern Australia and New Zealand (Heemstra 2016). It is easily distinguished from its congeners by the presence of numerous minute spinules on the dorsal- and anal-fin rays, strongly adherent ctenoid scales in adults and strong dorsaland anal-fin spines (James et al. 1988). Allocyttus guineensis from the southeastern Atlantic coast of Africa from Mauritania to Angola (Heemstra 2016) differs from congeners in the combination of strongly adherent cycloid scales and relatively weak dorsal- and anal-fin spines (Trunov 1982). Although the documented distributions of A. folletti and A. verrucosus have been confused due to incorrect iden- 
tifications, the former has been reported from the North Pacific (e.g., Myers 1960; Mecklenburg et al. 2002; present study) while the latter is currently thought to occur in the temperate waters of the North Atlantic and circumglobally in the Southern Hemisphere (e.g., Bray 2015; present study). Myers (1960) noted that $A$. folletti differs from A. verrucosus in having somewhat deciduous cycloid scales on the entire mid-lateral side area, lateral line and caudal peduncle, as well as more subtle features [more concave predorsal profile, flatter belly, less smoothly rounded snout, and more prominent and more rugose nasal boss (=bony projection)]. He also noted that none of the three type specimens of $A$. folletti from the coast of California had any trace of abdominal scutes, unlike in $A$. verrucosus.

Many oreo species are commercially important, including the one (Fig. 1A) exploited by boats fishing on the Emperor Seamounts, a volcanic seamounts chain extending from the western end of the Hawaiian Ridge $\left(30^{\circ} \mathrm{N}, 175^{\circ} \mathrm{E}\right)$ to the Aleutian Trench $\left(53^{\circ} \mathrm{N}, 164^{\circ} \mathrm{E}\right)$ in the western North Pacific (Nishida et al. 2016). However, the taxonomic identity of this species has been uncertain, due in part to confusion surrounding the taxonomy of the oreosomatid species known from the seas around Japan. Abe and Hotta (1962) reported and described a single oreosomatid specimen (181 mm SL) captured off Kinkazan, Miyagi Prefecture, Japan, and identified it as Allocyttus verrucosus rather than $A$. folletti, mainly because the specimen had two rows of scutes between the pectoral fin and preanal contour. Kobayashi et al. (1968) reported an "immature" specimen ( $268 \mathrm{~mm} \mathrm{SL}$ ) from the north-western Bering Sea and two "young" specimens (both $78 \mathrm{~mm} \mathrm{SL}$ ) from the central northern Pacific, and documented all three as A. verrucosus. They considered $A$. folletti a junior synonym of $A$. verrucosus, ascribing the distinguishing characters proposed by Myers (1960) to ontogenetic change or individual variance. Maruyama (1970) described a specimen from off Erimo (Pacific coast of Hokkaido, Japan) as A. verrucosus. Kido (1983) described a specimen $(292 \mathrm{~mm} \mathrm{SL}$, but the picture represents a different specimen of $431 \mathrm{~mm} \mathrm{SL}$ ) from off north-eastern Japan as $A$. verrucosus based on the two rows of scutes between the pectoral and pelvic fins.

In a revision of the oreosomatid fishes from the southern oceans, James et al. (1988) discussed that the records of A. verrucosus from the North Pacific (Welander et al. 1957; Abe and Hotta 1962; Kobayashi et al. 1968; Hughes 1981), as well as A. folletti in Myers (1960), represent a different taxon or taxa from A. verrucosus of the southern oceans (Fig. 1B) noting that "scale structure, scute patterns, and meristic counts ... together with the geographic separation suggest strongly that the northern and southern Pacific forms are not conspecific." However, James et al. (1988) did not adequately demonstrate how A. verrucosus and the North Pacific oreosomatid differ, and thus their comments were largely overlooked by subsequent researchers. Thereafter, the name A. verrucosus has been applied to the oreosomatid from the western North Pacific by many authors, mainly from Japan (Ida et al. 1992; Nakabo 1993, 2000, 2002; Amaoka et al. 1995, 2011; Inada 1997; Sheiko and Fedorov 2000; Maeda
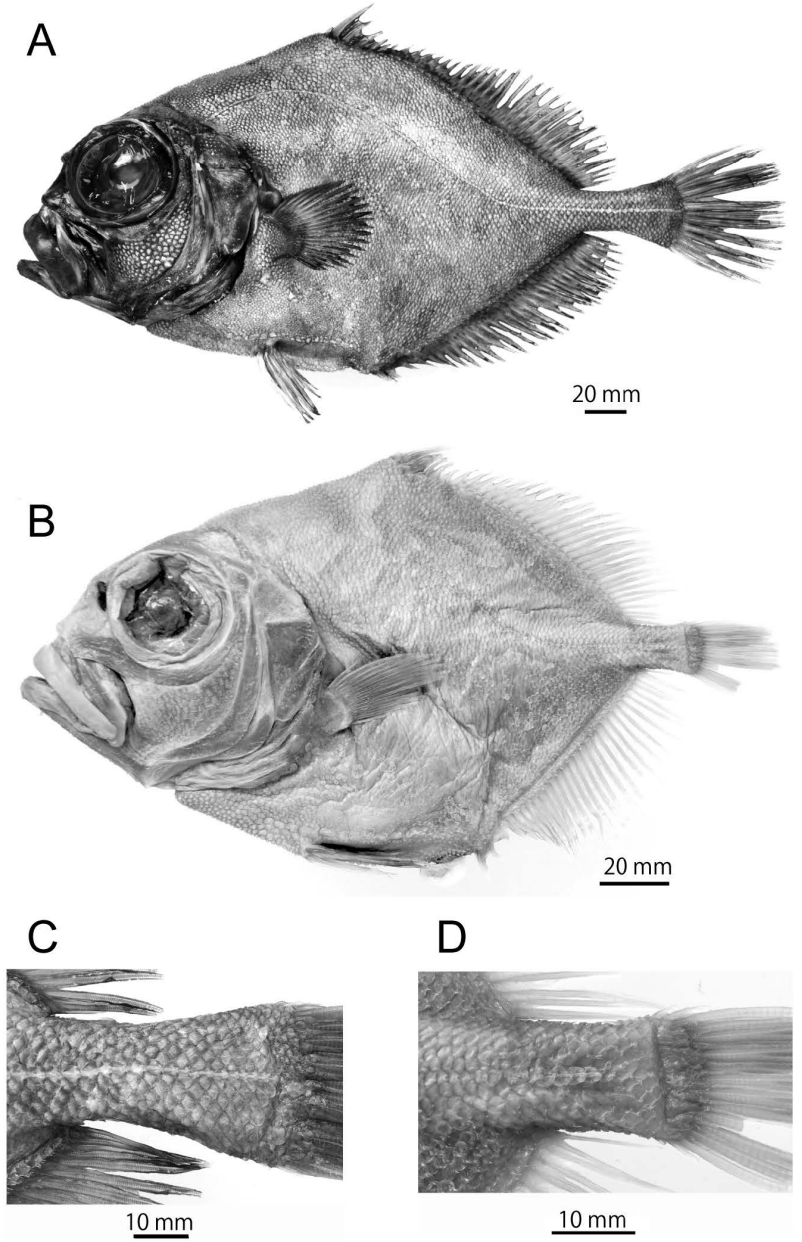

E

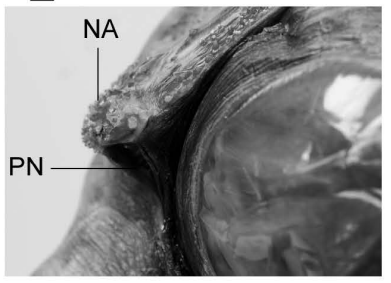

$10 \mathrm{~mm}$

F

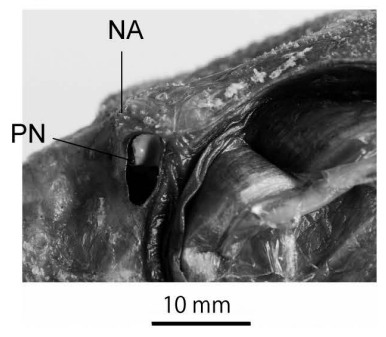

Fig. 1. Lateral view of oreosomatids. A, Allocyttus folletti from the Emperor Seamounts, SNFR 22402, $289.8 \mathrm{~mm}$ SL; B, Allocyttus verrucosus from New Zealand, NSMT-P 41168, $187.2 \mathrm{~mm}$ SL; caudal peduncle of A. folletti; C, SNFR 10560, $293.4 \mathrm{~mm}$ SL, Emperor Seamounts, and that of A. verrucosus; D, NSMT-P 41168, $187.2 \mathrm{~mm}$ SL, New Zealand; nasal of oreosomatids; E, A. folletti, SNFR 10561, 347 mm SL, Emperor Seamounts; F, A. verrucosus, NSMT-P 113107, $238.4 \mathrm{~mm}$ SL, west coast of Australia. Abbreviations: NA, nasal; PN, posterior nostril.

and Tsutsui 2003; Tokranov et al. 2004). One notable exception was Mecklenburg et al. (2002), who recorded the range of $A$. folletti as including the Bering Sea and the Pacific Ocean from central California to Honshu, Japan, and briefly noted that $A$. verrucosus of the South Pacific differed by having fewer fin elements and vertebrae, more cones (=scutes) in the upper abdominal row, and larger spots on the prejuvenile. Nakabo and Kai (2013) applied the name A. folletti to the oreosomatid of the western North Pacific, citing the discussion of James et al. (1988) and Mecklenburg et al. (2002), 
but did not document the differences between A. folletti and A. verrucosus. Mundy (2005) regarded the oreosomatid previously reported from the Emperor Seamounts (e.g., Borets 1986) as A. folletti without discussion.

At present, the differences between $A$. folletti and $A$. verrucosus are not well understood. For example, the presence or absence of two rows of scutes on the side of body between the pectoral and pelvic fins was considered as a valid character to distinguish $A$. verrucosus (present) from $A$. folletti (absent) (e.g., Myers 1960; Abe and Hotta 1962; Kido 1983). However, the scutes are illustrated in the figures of A. folletti in Mecklenburg et al. (2002), and the presence of the scutes was listed as a diagnostic character of $A$. folletti by Nakabo and Kai (2013), without discussion regarding the disagreement this posed with preceding studies (e.g., Myers 1960; Abe and Hotta 1962; Kido 1983). Ward et al. (1996) listed characters to distinguish the two species that had not been previously documented (number of pectoral-fin rays and lachrymal width) but they were not cited in the subsequent studies providing morphological data of $A$. folletti (e.g., Mecklenburg et al. 2002).

Uncertainty in the identity and applicable scientific name of the oreosomatid from the Emperor Seamounts will undermine the quality of future stock management, which is an international responsibility under the North Pacific Fisheries Commission (NPFC) (Nishida et al. 2016). This species is expected to be susceptible to over-exploitation (see Discussion), and the needs are therefore urgent to determine its scientific name and implement effective management measures.

The major goal of this study is to establish the taxonomic identity of the oreosomatid from the Emperor Seamounts, thereby contributing to appropriate stock management, and to provide reliable diagnostic characters to distinguish $A$. folletti from A. verrucosus.

\section{Materials and Methods}

The left-hand side of each specimen was examined except when damaged or in an abnormal condition. Methods of counts and measurements mostly follow James et al. (1988), with some modifications. For standard length (SL), head length (HL), predorsal-fin length (PDFL), and preanal-fin length (PAFL), both the ordinary measurement from the snout tip (abbreviated as SL1, HL1, PDFL1 and PAFL1 respectively), and a modified measurement from the anterior tip of maxillary (SL2, HL2, PDFL2 and PAFL2 respectively), were made. The latter is thought to provide a more accurate basis for comparative proportions because it eliminates the influence of any mouth protrusion, but the former measurements are needed for comparisons with the literature. SL2 and HL2 correspond to Myers' (1960) "standard length minus premaxillary" and "head length minus premaxillary" respectively. The mouth, if protruding, was retracted to its natural closed position when possible; if not, the specimen was excluded for measurements from the snout tip to preclude any biased values. For body depth (BD), BD1 is the greatest vertical dimension of body at the dorsal-fin origin (as in James et al. 1988), and BD2 is the distance from the dorsal-fin origin to the anal-fin origin (as in Myers 1960). Abdominal edge length (AEL) and thoracic edge length (TEL) follow Myers (1960). P2O-AFO is the length between the pelvic-fin origin and the anal-fin origin [=thoracic edge length of James et al. (1988)]. For caudal-peduncle length (CPL), CPL1 is the distance from the anal-fin insertion to the middle of the caudal-fin base; CPL2 is the distance from the anal-fin insertion to the exposed origin of the first ventral procurrent ray (as in Yearsley and Last 1998). Depth of caudal-fin base is the depth of the caudal-fin base between the exposed origins of the first procurrent rays of the upper and lower lobes. Lachrymal width (LW) is the minimum dorsoventral dimension of part of lachrymal below eye (posterior to the articulation with the lateral ethmoid) (Ward et al. 1996; G. K. Yearsley, pers. comm.). S-DFB and S-AFR are the serial enlarged scales at the dorsal- and analfin bases respectively. Vertebrae were examined by radiography. The vertebrae, S-DFB, S-AFB and the spines on S-DFB and S-AFB of the holotype of A. folletti (CAS-SU 15377) were counted from the digital images downloaded from "Ichthyology Primary Types Imagebase" of California Academy of Sciences (https://www.calacademy.org/scientists/ ichthyology-primary-types-imagebase). Institutional acronyms follow Fricke and Eschmeyer (2019).

Comparative material examined. Allocyttus folletti. 2 specimens, Japan. NSMT-P $30689(\mathrm{n}=1), 194.7 \mathrm{~mm}$ SL, Suruga Bay, Shizuoka Prefecture, $34^{\circ} 50^{\prime} \mathrm{N}, 138^{\circ} 35^{\prime} \mathrm{E}$, July-August 1969; HUMZ $72625(\mathrm{n}=1), 287.1 \mathrm{~mm} \mathrm{SL}$, off Fukushima Prefecture, $37^{\circ} 30.0^{\prime} \mathrm{N}, 142^{\circ} 19.5^{\prime} \mathrm{E}, 815-860 \mathrm{~m}$ deep, 24 January 1978, trawl. Allocyttus verrucosus. 7 specimens. NSMT-P $41168(\mathrm{n}=1), 187.2 \mathrm{~mm}$ SL, New Zealand, date unknown, R/V Shinkai-maru; NSMT-P 112857 $(\mathrm{n}=1), 201.5 \mathrm{~mm}$ SL, West coast of Australia, date unknown, R/V Kaiyo-maru; NSMT-P 112858, 110.2 mm SL, West coast of Australia, date unknown, R/V Kaiyo-maru; NSMT-P $113107(\mathrm{n}=1), 238.4 \mathrm{~mm}$ SL, West coast of Australia, $31^{\circ} 39.3^{\prime} \mathrm{S}, 114^{\circ} 44.0^{\prime} \mathrm{E}, 1035 \mathrm{~m}$ deep, 15 November 1975, R/V Kaiyo-maru. BSKU 48476-48478 ( $n=3), 121.4-$ $151.3 \mathrm{~mm} \mathrm{SL}$, off South Africa, $34^{\circ} 26.6^{\prime} \mathrm{S}, 26^{\circ} 00.8^{\prime} \mathrm{E}, 750 \mathrm{~m}$, 3 June 1990, bottom trawl.

\section{Results}

\section{Allocyttus folletti Myers, 1960}

(Figs 1A, C, E, 2A, C, 3)

Allocyttus verrucosus (non Gilchrist, 1906): Welander et al. 1957: 245-246 (description; off North Pacific); Abe and Hotta 1962: 152-156, figs 1-8 (description; off Kinkazan, Japan); Kobayashi et al. 1968: 1-5, pl. I (description; western Bering Sea and western North Pacific); Maruyama 1970: 52-54, fig. 9 (description; off Erimo, Japan); Maruyama 1971: 33, fig. 36 (list; off Erimo, Japan); Kido 1983: 126-127, 203, fig. (description: Japan); Machida 1984: 118, pl. 103-A (description; Japan); Ida et al. 1992: 86, fig. (description; off Iwate Pref., Japan); Nakabo 1993: 
467, 3 figs (pictorial key); Amaoka et al. 1995: 127, fig. (description; Hokkaido, Japan); Inada 1997: 168, fig. (note on distribution; off Kushiro, Japan); Nakabo 2000: 508, 3 figs. (pictorial key); Nakabo 2002: 508, 3 figs (pictorial key); Tokranov et al. 2004: 210-212, figs 1-3 (distribution, length-weight relationship; Northern Kurile Islands and Southeast Kamchatka); Kitagawa et al. 2008: 50, fig. (diagnosis; Japan); Amaoka et al. 2011: 168, fig. (description; Hokkaido, Japan).

Allocyttus folletti Myers, 1960: 89-98, fig. 1 (original description; type locality: California); Miller and Lea 1972: 84-85, fig. (diagnosis); Anderson et al. 1979: 262 (meristics; California); Eschmeyer and Herald 1983: 126, pl. 48, fig. 18 (diagnosis); Nagtegaal 1983: 89, fig. 1 (description; British Columbia); Cook and Long 1985: 57 (occurrence; Bering Sea); Gillespie and Saunders 1994: 348 (description; British Columbia); Ward et al. 1996: 41 (diagnosis); Mecklenburg et al. 2002: 327-329, 2 figs (diagnosis; Alaska); Nakabo and Kai 2013: 601, 1902, 3 figs (pictorial key, taxonomic note); Kamikawa 2017: 176, fig. (description; West Coast of U. S. A.); Amaoka et al. 2020: 207, figs (description; Hokkaido, Japan).

Allocyttus sp.: Hart 1973; 266-267, fig. (description of juvenile; mid-Pacific, west of British Columbia).

Material examined. Emperor Seamounts-21 specimens. SNFR $10560(\mathrm{n}=1), 293.4 \mathrm{~mm}$ SL, Koko Seamount, $35^{\circ} 39^{\prime} \mathrm{N}, \quad 171^{\circ} 03^{\prime} \mathrm{E}$ to $35^{\circ} 38^{\prime} \mathrm{N}, 171^{\circ} 03^{\prime} \mathrm{E}, \quad 361-355 \mathrm{~m}$ deep, 24 July 2007, F/V Tomi-maru No. 58; SNFR 10561 $(\mathrm{n}=4), \quad 301-347 \mathrm{~mm}$ SL, collected with SNFR 10560;
SNFR $11127(\mathrm{n}=1), 279.4 \mathrm{~mm}$ SL, Kinmei Seamount, $33^{\circ} 40.0^{\prime} \mathrm{N}, 172^{\circ} 00.0^{\prime} \mathrm{E}, 600 \mathrm{~m}, 13$ May 1994; SNFR 18692 $(\mathrm{n}=1), 537 \mathrm{~mm}$ SL, Kinmei Seamount, $33^{\circ} 46^{\prime} \mathrm{N}, 171^{\circ} 54^{\prime} \mathrm{E}$, 850-893 m, 27 September 2012, F/V Shoshin-maru No. 88; SNFR 22400-22408 ( $\mathrm{n}=9), 269.3-300.8 \mathrm{~mm} \mathrm{SL}$, Yuryaku Seamount, $32^{\circ} 19.16^{\prime} \mathrm{N}, \quad 172^{\circ} 19.25^{\prime} \mathrm{E}$ to $32^{\circ} 40.63^{\prime} \mathrm{N}$, $172^{\circ} 13.09^{\prime} \mathrm{E}, 486-670 \mathrm{~m}, 5$ February 2019, F/V Kaiyo-maru No. 38; NSMT-P $72807(\mathrm{n}=1), 337 \mathrm{~mm}$ SL, Colahan Seamount, $31^{\circ} 01^{\prime} \mathrm{N}, 175^{\circ} 54^{\prime} \mathrm{E}$ to $31^{\circ} 02^{\prime} \mathrm{N}, 175^{\circ} 52^{\prime} \mathrm{E}, 285 \mathrm{~m}$, 17 September 2005, F/V. Yokei-maru No. 5; FAKU72487 $(\mathrm{n}=1), 328 \mathrm{~mm}$ SL, Kinmei Seamount, $33^{\circ} 40^{\prime} \mathrm{N}, 172^{\circ} 00^{\prime} \mathrm{E}$, $600 \mathrm{~m}, 13$ May 1994; FAKU72575 ( $\mathrm{n}=1), 397 \mathrm{~mm}$ SL, collected with FAKU72487; FAKU119906 $(\mathrm{n}=1), 321 \mathrm{~mm}$ SL, Hancock Seamounts, 3 March 1973, R/V Kaiyomaru; FAKU119907 $(\mathrm{n}=1), 382 \mathrm{~mm}$ SL, collected with FAKU119906.

Description. Meristics are presented in Table 1, and morphometrics as \% of SL1 and SL2 are presented in Tables 2 and 3 respectively. Body oval, laterally compressed and deep; deepest at dorsal-fin origin, its depth greater than head length. Predorsal profile usually slightly convex (but sometimes straight) from dorsal-fin origin to vertical through uppermost end of gill opening, slightly concave or straight in front of same vertical. Snout profile steep. Thorax flat ventrally. Midline of abdomen gently keeled between pelvic-fin origin and anus (abdominal edge). Caudal peduncle slender, deeper posteriorly (Fig. 1C), deepest at caudalfin base.

Head large, $34.6-39.3 \%$ of SL1. Eye large, $12.5-18.4 \%$ of SL1. Mouth large, oblique, greatly extensible. Maxillary ex-

Table 1. Meristics of Allocyttus folletti and A. verrucosus.

\begin{tabular}{|c|c|c|c|c|c|}
\hline \multirow[b]{2}{*}{ Characters } & \multicolumn{3}{|c|}{ A. folletti } & \multicolumn{2}{|c|}{ A. verrucosus } \\
\hline & $\begin{array}{l}\text { Emperor Seamounts } \\
\qquad \mathrm{N}=21\end{array}$ & $\begin{array}{l}\text { Japan } \\
\mathrm{N}=2\end{array}$ & $\begin{array}{l}\text { Myers }(1960)^{1}+\text { holotype image } \\
\qquad \mathrm{N}=3\end{array}$ & $\begin{array}{l}\text { Examined specimens } \\
\qquad \mathrm{N}=7\end{array}$ & $\begin{array}{l}\text { Gilchrist (1906) } \\
\qquad \mathrm{N}=1 ?\end{array}$ \\
\hline DS & $6-7$ & 6 & $7(5-7)$ & 6 & 6 \\
\hline $\mathrm{DR}$ & $30-33$ & 32 & $32(30-33)$ & $28-30$ & 31 \\
\hline $\mathrm{DS}+\mathrm{DR}$ & $37-40$ & 38 & $39(37-39)$ & $34-36$ & 37 \\
\hline AS & $2-4$ & $2-3$ & $3(3)$ & $2-3$ & 3 \\
\hline $\mathrm{AR}$ & $29-32$ & $30-31$ & $31(31-32)$ & $27-29$ & 29 \\
\hline $\mathrm{AS}+\mathrm{AR}$ & $32-35$ & $32-34$ & $34(34-35)$ & $29-31$ & 32 \\
\hline P1R & $19-21$ & $19-21$ & $21(19-21)$ & $17-20$ & - \\
\hline $\mathrm{P} 2 \mathrm{~S}$ & 1 & 1 & $1(1)$ & 1 & - \\
\hline P2R & 6 & 6 & $6(6)$ & 6 & - \\
\hline LLS & $82-93$ & $88-90$ & 95 (85-95) & $82-92$ & 95 \\
\hline UGR & $3-6$ & $4-6$ & $6(6-7)$ & $3-6$ & - \\
\hline LGR & $16-22$ & $19-20$ & $19(19-25)$ & $18-19$ & - \\
\hline TGR & $20-27$ & $23-26$ & $25(25-32)$ & $21-24$ & - \\
\hline AV & $12-13$ & $12-13$ & 12 & $12-13$ & - \\
\hline $\mathrm{CV}$ & $24-27$ & $27-28$ & 28 & $23-25$ & - \\
\hline TV & $37-40$ & 40 & 40 & $35-37$ & - \\
\hline S-DFB & $31-42$ & $32-33$ & 33 & $26-31$ & - \\
\hline S-AFB & $29-37$ & $29-31$ & 29 & $25-28$ & - \\
\hline
\end{tabular}

Abbreviations: AR, anal-fin rays; AS, anal-fin spines; AV, abdominal vertebrae; CV, caudal vertebrae; DR, dorsal-fin rays; DS, dorsal-fin spines; LGR, gill rakers of lower limb; LLS, lateral-line scales; P1R, pectoral-fin rays; P2R, pelvic-fin rays; P2S, pelvic-fin spine; S-AFB, enlarged scales of anal-fin base; S-DFB, enlarged scales of dorsal-fin base; TGR, total gill rakers; TV, total vertebrae; UGR, gill rakers of upper limb. ${ }^{1}$ Data of the holotype, followed by the range of holotype + two paratypes in parentheses. Digits in italics were counted from the digital images of the holotype (CAS-SU 15377). 
Table 2. Morphometrics as \% of SL1 (snout tip to middle of caudal-fin base) of Allocyttus folletti and A. verrucosus.

\begin{tabular}{|c|c|c|c|c|c|}
\hline \multirow[b]{2}{*}{ Characters } & \multicolumn{3}{|c|}{ A. folletti } & \multicolumn{2}{|c|}{ A. verrucosus } \\
\hline & $\begin{array}{l}\text { Emperor Seamounts } \\
\qquad \mathrm{N}=21\end{array}$ & $\begin{array}{l}\text { Japan } \\
\mathrm{N}=1\end{array}$ & $\begin{array}{c}\text { Myers }(1960)^{1} \\
\quad \mathrm{~N}=3\end{array}$ & $\begin{array}{l}\text { Examined specimens } \\
\qquad \mathrm{N}=7\end{array}$ & Gilchrist (1906) \\
\hline SL1 (mm) & $269.3-537$ & 287.1 & $347(162-347)$ & $110.2-238.4$ & $?$ \\
\hline HL1 & $34.6-39.3$ & 34.6 & $36.3(36.3-42.0)$ & $40.2-43.1$ & 41.5 \\
\hline PDFL1 & $50.2-57.6$ & 53.5 & $51.0(51.0-58.0)$ & $53.0-62.3$ & 60.8 \\
\hline PAFL1 & $53.8-62.4$ & 55.5 & $62.8(62.8-63.6)$ & $66.5-82.0$ & 64.5 \\
\hline BD1 & $50.4-55.9$ & 53.3 & $51.3(-)$ & $56.9-68.7$ & 64.1 \\
\hline BD2 & $51.5-57.5$ & 53.7 & $53.3(53.3-58.6)$ & $57.0-65.7$ & 65.2 \\
\hline DFBL & $42.8-50.9$ & 47.7 & $49.3(46.0-49.3)$ & $43.9-48.1$ & 48.7 \\
\hline AFBL & $38.8-43.1$ & 43.8 & $40.1(37.8-42.0)$ & $36.8-40.5$ & 41.4 \\
\hline HW & $18.7-22.3$ & 19.8 & - & $19.8-23.3$ & - \\
\hline UJL & $14.0-17.0$ & 15.2 & $15.9(15.4-17.9)$ & $14.7-19.2$ & 18.3 \\
\hline LJL & $19.8-22.7$ & 21.5 & $19.6(19.6-23.5)$ & $22.1-24.4$ & 23.5 \\
\hline ED & $12.5-18.4$ & 16.3 & $14.2(-)$ & $15.1-19.0$ & 18.4 \\
\hline SNL & $8.5-13.6$ & 9.6 & $10.1(10.1-12.4)$ & $10.3-13.5$ & 13.0 \\
\hline LW & $1.7-2.5$ & 2.1 & 2.0 & $2.5-3.6$ & 4.2 \\
\hline P1L & $14.5-17.6$ & 15.3 & $16.4(15.4-19.1)$ & $16.1-19.9$ & 18.4 \\
\hline P2L & $14.2-19.3$ & 17.1 & - & $19.0-24.4$ & 19.1 \\
\hline DS2L & $8.4-11.0$ & 10.2 & $8.2(8.2-11.7)$ & $7.4-11.6$ & 7.9 \\
\hline DRL & $11.6-14.7$ & 13.0 & $11.8(10.8-13.6)$ & $14.6-17.3$ & 18.2 \\
\hline AS1L & $5.2-7.6$ & - & - & $5.9-6.9$ & 6.4 \\
\hline ARL & $13.9-15.5$ & 14.0 & $13.3(12.7-16.1)$ & $15.5-18.3$ & 18.5 \\
\hline P2SL & $12.9-16.0$ & 15.2 & $13.0(13.0-22.2)$ & $11.0-16.7$ & - \\
\hline TEL & $14.6-18.5$ & 16.6 & $17.3(17.3-18.5)$ & $20.6-24.9$ & - \\
\hline AEL & $14.6-20.2$ & 18.2 & $18.4(18.4-19.7)$ & $20.1-26.0$ & - \\
\hline P2O-AFO & $17.7-23.1$ & 20.5 & - & $23.9-29.6$ & - \\
\hline CPL1 & $11.2-14.8$ & 14.6 & $13.5(-)$ & $7.7-10.2$ & 11.9 \\
\hline CPL2 & $10.4-13.0$ & 13.8 & - & $6.1-8.9$ & - \\
\hline $\mathrm{CPD}$ & $5.0-6.7$ & 6.3 & $5.8(5.8-6.2)$ & $5.9-6.9$ & 7.0 \\
\hline
\end{tabular}

Abbreviations: AEL, abdominal edge length; AFBL, anal-fin base length; ARL, longest anal-fin ray length; AS1L, 1st anal-fin spine length; BD1, body depth 1; BD2, body depth 2; CPD, caudal-peduncle depth; CPL1, caudal peduncle length 1; CPL2, caudal-peduncle length 2; DFBL, dorsalfin base length; DRL, longest dorsal-fin ray length; DS2L, 2nd dorsal-fin spine length; ED, eye diameter; HL1, head length 1; HW, head width; LJL, lower-jaw length; LW, lachrymal width; P1L, pectoral-fin length; P2L, pelvic-fin length; P2SL, pelvic-fin spine length; PAFL1, pre-anal fin length 1; PDFL1, pre-dorsal fin length 1; P2O-AFO, length from pelvic-fin origin to anal-fin origin; SNL, snout length; TEL, thoracic edge length. For methods of measurement, refer to text. ${ }^{1}$ Data of holotype, and range of holotype + two paratypes in parentheses. Digits in italics indicate measurement from figure of original description.

tending posteriorly nearly to below middle of eye when mouth closed. Opercular bones with radiated bony striations, scaleless, except opercle sparsely scaled. Numerous spinules on all exposed bones on head. Nasal projected anteriorly above anterior nostril, with enlarged spinules (more prominent in larger specimens). Nostrils close together: anterior one circular, oriented anteriorly; posterior one vertically elongate, oriented laterally, immediately anterior to eye. Nasal projected forward above posterior nostril (Fig. 1E). Lower surface of dentary with numerous spinules and longitudinal striations. Teeth on both jaws minute, conical, slightly curved inward, in irregular 1-3 rows. Vomerine teeth similar to jaw teeth in shape, size and arrangement. Palatine teeth absent.

Dorsal-, anal-, and pelvic-fin spines robust, densely striated; first dorsal-fin spine about one-third of second spine in length. First anal-fin spine longer than second and third spines. All dorsal-, anal- and pectoral-fin soft rays simple, densely segmented. Spinous and soft-ray portions of anal fin continuous, with low membrane. Pectoral fin small, round- ed, posterior margin falling slightly short of vertical through anal-fin origin. Pelvic fin reaching or extending slightly beyond anus when depressed, its spine reaching or falling slightly short of anus. Caudal fin rounded.

Lateral line gently arched, nearly parallel to dorsal contour in anterior two-thirds, posterior third straight. Scales mostly cycloid and deciduous mid-laterally (Fig. 2A), and on caudal peduncle, basal part of caudal fin and lateral line; thickened cycloid or ctenoid with a few spinules on lower side of thorax and abdomen; thickened ctenoid with prominently enlarged and elevated spinules at nape and ventral surface of thorax and abdomen; those on ventral midline of abdomen especially thickened and enlarged in large specimens; row of enlarged scales along base of dorsal and anal fins (S-DFB and S-AFB respectively) forming sheath of scales, with up to 7-12 (margin) and 1-3 (center) elevated spines per scale (Fig. 2C). Single row of smaller scales with 1-3 spinules along medial side of S-DFB and S-AFB. Two nearly horizontal rows of scutes (assemblage of somewhat enlarged scales) on abdomen between pectoral and pel- 
Table 3. Morphometrics as \% of SL2 (anterior tip of maxillary to middle of caudal-fin base) of Allocyttus folletti and A. verrucosus.

\begin{tabular}{|c|c|c|c|c|c|}
\hline \multirow[b]{2}{*}{ Characters } & \multicolumn{3}{|c|}{ A. folletti } & \multicolumn{2}{|c|}{ A. verrucosus } \\
\hline & $\begin{array}{l}\text { Emperor Seamounts } \\
\qquad \mathrm{N}=21\end{array}$ & $\begin{array}{l}\text { Japan } \\
\mathrm{N}=2\end{array}$ & $\begin{array}{l}\text { Myers }(1960)^{1} \\
\quad \mathrm{~N}=3\end{array}$ & $\begin{array}{l}\text { Examined specimens } \\
\qquad \mathrm{N}=7\end{array}$ & Gilchrist (1906) \\
\hline SL2 (mm) & $264.7-526$ & $187.4-286.1$ & $341(160-341)$ & $107.4-232.8$ & $?$ \\
\hline HL2 & $32.8-37.1$ & $33.8-38.0$ & $35.8(35.8-41.3)$ & $38.8-40.8$ & 39.0 \\
\hline PDFL2 & $49.1-57.2$ & $51.3-53.7$ & 50.1 & $51.2-60.9$ & 57.4 \\
\hline PAFL2 & $55.5-64.5$ & $56.7-69.1$ & 62.8 & $68.5-80.5$ & 66.1 \\
\hline BD1 & $51.2-56.6$ & $53.4-56.1$ & 51.8 & $59.5-69.4$ & 66.1 \\
\hline BD2 & $52.3-58.7$ & $53.9-56.6$ & $54.3(54.3-59.4)$ & $59.2-66.4$ & 67.2 \\
\hline DFBL & $44.4-51.0$ & $44.8-47.9$ & $50.2(47.2-50.2)$ & $45.1-49.3$ & 50.2 \\
\hline AFBL & $39.4-43.7$ & $39.2-43.9$ & $40.8(38.9-42.5)$ & $37.7-41.7$ & 42.6 \\
\hline HW & $19.3-22.6$ & $19.9-21.7$ & - & $20.3-23.6$ & - \\
\hline UJL & $14.3-17.0$ & 15.2 & $16.1(15.9-18.1)$ & $15.1-19.4$ & 18.9 \\
\hline LJL & $20.2-22.7$ & 21.6 & $19.9(19.9-23.8)$ & $22.7-25.5$ & 24.2 \\
\hline ED & $12.7-18.7$ & $16.4-18.4$ & $14.5(-)$ & $15.4-19.2$ & 19.0 \\
\hline SNL & $8.6-14.2$ & 9.6 & $10.3(10.3-12.5)$ & $10.4-13.9$ & 13.5 \\
\hline LW & $1.7-2.5$ & $1.8-2.1$ & 2.1 & $2.5-3.7$ & 4.3 \\
\hline P1L & $14.5-17.8$ & $15.3-15.4$ & $16.7(15.9-19.4)$ & $16.5-20.6$ & 19.0 \\
\hline $\mathrm{P} 2 \mathrm{~L}$ & $14.5-19.5$ & $17.1-19.7$ & - & $19.4-24.6$ & 19.7 \\
\hline DS2L & $8.5-11.1$ & $10.2-10.8$ & $8.4(8.4-11.9)$ & $7.6-11.9$ & 8.2 \\
\hline DRL & $11.8-15.0$ & $13.1-14.7$ & $12.0(11.1-13.8)$ & $15.0-18.0$ & 18.8 \\
\hline AS1L & $5.3-7.7$ & 6.9 & - & $6.1-7.1$ & 6.6 \\
\hline ARL & $14.0-15.7$ & $14.1-16.8$ & $13.5(13.1-16.3)$ & $15.9-18.7$ & 19.1 \\
\hline P2SL & $11.4-16.3$ & $15.3-16.7$ & $13.2(13.2-22.5)$ & $11.3-16.9$ & - \\
\hline TEL & $15.2-18.7$ & $16.6-18.0$ & $17.6(17.6-19.1)$ & $21.1-25.1$ & - \\
\hline AEL & $14.7-20.4$ & $18.2-18.7$ & $18.8(18.8-20.2)$ & $20.9-26.7$ & \\
\hline P2O-AFO & $18.0-23.4$ & $20.6-21.3$ & - & $23.7-30.3$ & - \\
\hline CPL1 & $11.3-15.1$ & $12.4-14.7$ & 13.5 & $7.9-10.6$ & 12.3 \\
\hline CPL2 & $10.5-13.2$ & $11.2-13.9$ & - & $6.3-9.3$ & - \\
\hline CPD & $5.0-6.8$ & $5.7-6.3$ & $5.9(5.9-6.3)$ & $6.1-7.0$ & 7.2 \\
\hline
\end{tabular}

Abbreviations: AEL, abdominal edge length; AFBL, anal-fin base length; ARL, longest anal-fin ray length; AS1L, 1st anal-fin spine length; BD1, body depth 1; BD2, body depth 2; CPD, caudal-peduncle depth; CPL1, caudal peduncle length 1; CPL2, caudal-peduncle length 2; DFBL, dorsalfin base length; DRL, longest dorsal-fin ray length; DS2L, 2nd dorsal-fin spine length; ED, eye diameter; HL2, head length 2; HW, head width; LJL, lower-jaw length; LW, lachrymal width; P1L, pectoral-fin length; P2L, pelvic-fin length; P2SL, pelvic-fin spine length; PAFL2, pre-anal fin length 2; PDFL2, pre-dorsal fin length 2; P2O-AFO, length from pelvic-fin origin to anal-fin origin; SNL, snout length; TEL, thoracic edge length. For methods of measurements, refer to text. ${ }^{1}$ Data of holotype, and range of holotype + two paratypes in parentheses. Digits in italics indicating measurements from figures of original description.

vic fins; in upper row enlarged scales forming 1-5 patches, weak or dislodged in some specimens; in lower row, enlarged scales forming continuous row (Fig. 3A). Body uniformly dark brown or grayish brown, fins darker; cheek and opercles blueish.

Distribution. Emperor Seamounts (Mundy 2005; present study), Bering Sea and Pacific Ocean east to Central California and west to Japan (from Hokkaido to Ibaraki Prefecture) (Mecklenburg et al. 2002; Nakabo and Kai 2013).

\section{Discussion}

Identification. Four genera are recognized in the family Oreosomatidae (James et al. 1988; Yearsley and Last 1998): Pseudocyttus Gilchrist, 1906, Oreosoma Cuvier, 1829, Neocyttus Gilchrist, 1906, and Allocyttus McCulloch, 1914. The present specimens are identified as Allocyttus, being distinguished from the other genera by the following combination of characters (see James et al. 1988): from Pseudocyttus by having the first dorsal-fin spine shorter than the second (vs. longer in Pseudocyttus), and six pelvic-fin soft rays (vs. five); from Oreosoma by having radiating striations and lacking a horizontal ridge on the opercle (vs. lacking radiating striations and having a prominent horizontal ridge), and relatively small eye diameter (39-46\% vs. $52-60 \%$ of HL1); and from Neocyttus by having a nearly straight or slightly convex predorsal profile (vs. strongly concave and sharply risen).

Within the genus Allocyttus, the present specimens can be easily distinguished from A. niger (see James et al. 1988), in lacking spinules on the dorsal and anal-fin rays (vs. spinules present), fewer total gill rakers (20-27 vs. 26-33), and fewer lateral-line scales (82-93 vs. 91-102). From A. guineensis (see Trunov 1982; Heemstra 2016), the present specimens differ in having more anal-fin rays (29-32 vs. $26-27)$, a shorter head length (HL1: $34.6-39.3 \%$ vs. ca. $40-45 \%$ of SL1), a longer 2nd dorsal-fin spine (8.4-11.0\% vs. $4.6-5.7 \%$ of SL1) and a longer 1 st anal-fin spine $(5.2-7.6 \%$ vs. $1.7-$ $4.4 \%$ of SL1).

Comparisons of the present specimens with the holotype 
A

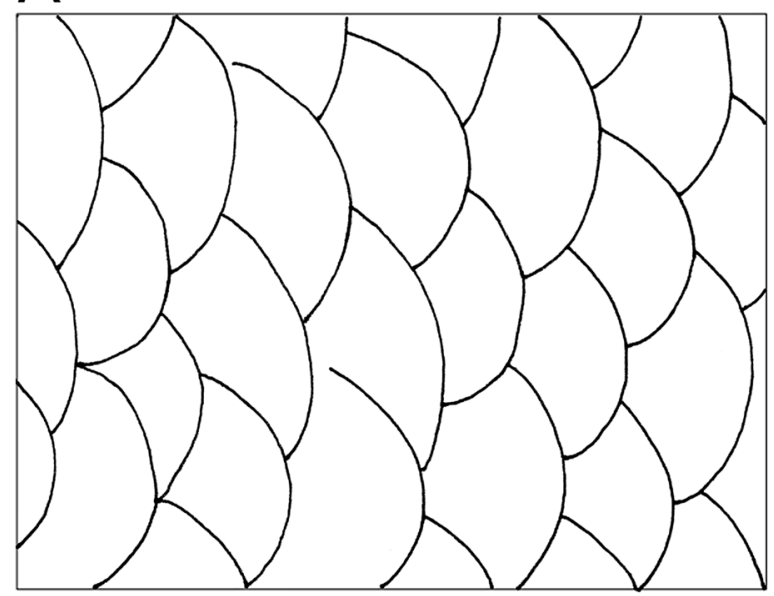

$5 \mathrm{~mm}$

C

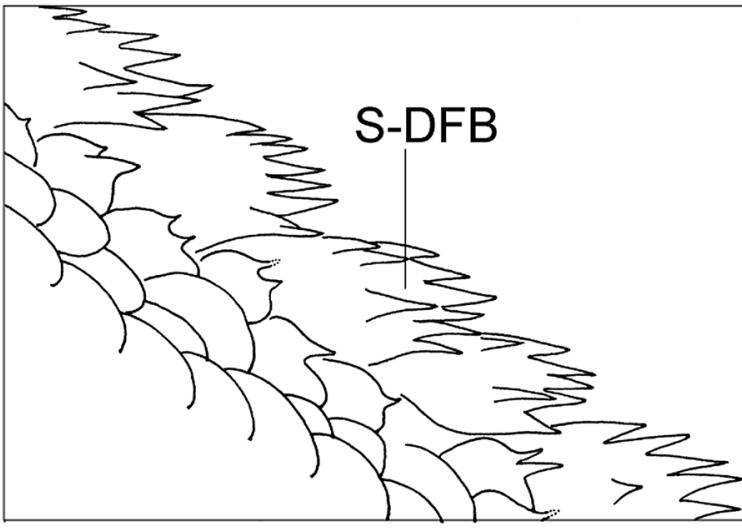

$5 \mathrm{~mm}$
B

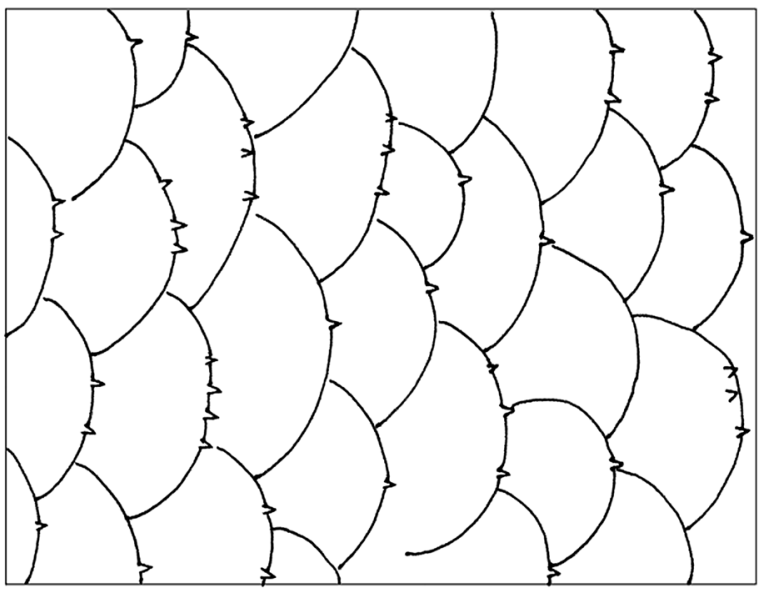

$5 \mathrm{~mm}$
D

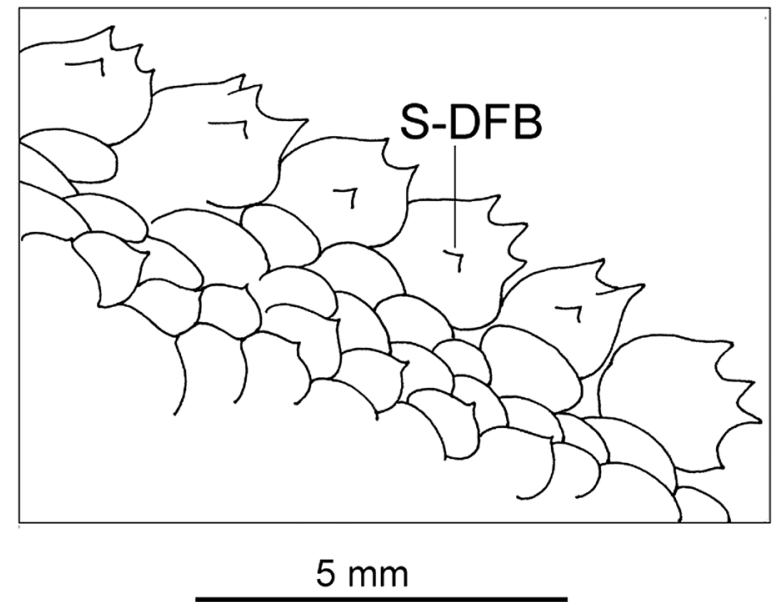

Fig. 2. Scales on mid-side of body in, (A) Allocyttus folletti, FAKU 72575, $397 \mathrm{~mm}$ SL, Emperor Seamounts, and (B) Allocyttus verrucosus, NSMT-P 113107, $238.4 \mathrm{~mm}$ SL, Australia; enlarged scales of dorsal-fin base (S-DFB) in (C) A. folletti, SNFR 22403, $289.3 \mathrm{~mm}$ SL, Emperor Seamounts, and (D) A. verrucosus, BSKU 48476, $136.5 \mathrm{~mm}$ SL, off South Africa.

of $A$. folletti and with A. verrucosus [comparative specimens, and one of the syntypes based on the illustration in Gilchrist (1906)] are presented in Tables 1-3. The ranges for most of the characters in the present specimens include the value for the holotype of $A$. folletti. However, there are many characters that disagree with the characters in A. verrucosus, including with respect to meristics (Table 1), more dorsaland anal-fins spines + rays (37-40 vs. $34-37$ and $32-35$ vs. 29-32 respectively), more total vertebrae (37-40 vs. $35-37$ ) and more S-DFB (31-42 vs. 26-31) and S-AFB (29-37 vs. 25-28). With respect to morphometrics (Tables 2, 3), the present specimens disagreed with A. verrucosus in having a smaller head (HL1: $34.6-39.3 \%$ vs. $40.2-43.1 \%$ of SL1; HL2: $32.8-38.0 \%$ vs. $38.8-40.8 \%$ of SL2), a shorter preanal-fin length (PAFL1: $53.8-62.4 \%$ vs. $64.5-82.0 \%$ of SL1; PAFL2: $55.5-69.1 \%$ vs. $68.5-80.5 \%$ of SL2), a shallower BD1 (50.4$55.9 \%$ vs. $56.9-68.7 \%$ of SL1; $51.2-56.6 \%$ vs. $59.5-69.4 \%$ of SL2), a narrower LW (1.7-2.5\% vs. $2.5-4.2 \%$ of SL1; $1.7-2.5 \%$ vs. $2.5-4.3 \%$ of SL2), a longer CPL1 (11.2-14.8\% vs. $7.7-11.9 \%$ of SL1; $11.3-15.1 \%$ vs. $7.9-12.3 \%$ of SL2) and CPL2 (10.4-13.8\% vs. $6.1-8.9 \%$ of SL1; $10.5-13.9 \%$ vs. $6.3-$ $9.6 \%$ of SL2), a shorter DRL (11.6-14.7\% vs. $14.6-18.2 \%$ of SL1; $11.8-15.0 \%$ vs. $15.0-18.8 \%$ of SL2), a shorter TEL (14.6-18.5\% vs. $20.6-24.9 \%$ of SL1; $15.2-18.7 \%$ vs. $21.1-$ $25.1 \%$ of SL2), a shorter AEL (14.6-20.2\% vs. $20.1-26.0 \%$ of SL1; $14.7-20.4 \%$ vs. $20.9-26.7 \%$ of SL2) and a shorter P2O-AFO (17.7-23.1\% vs. $23.9-29.6 \%$ of SL1; $18.0-23.4 \%$ vs. $23.7-30.3 \%$ of SL2). In addition, the caudal peduncle was deeper posteriorly (Fig. 1C, D) and CFD was $140-188 \%$ of $\mathrm{CPD}$ in the examined specimens, agreeing with the holotype of A. folletti (180\%) and greater than in A. verrucosus (111-127\% in comparative specimens).

The scales of the entire mid-lateral area, lateral line, and caudal peduncle of the examined specimens are cycloid, agreeing with the holotype of A. folletti (see Myers 1960), and differing from the ctenoid scales in $A$. verrucosus (James et al. 1988; present study) (Fig. 2A, B). In A. folletti, the maximum number of spines of S-DFB and S-AFB is 7-12 

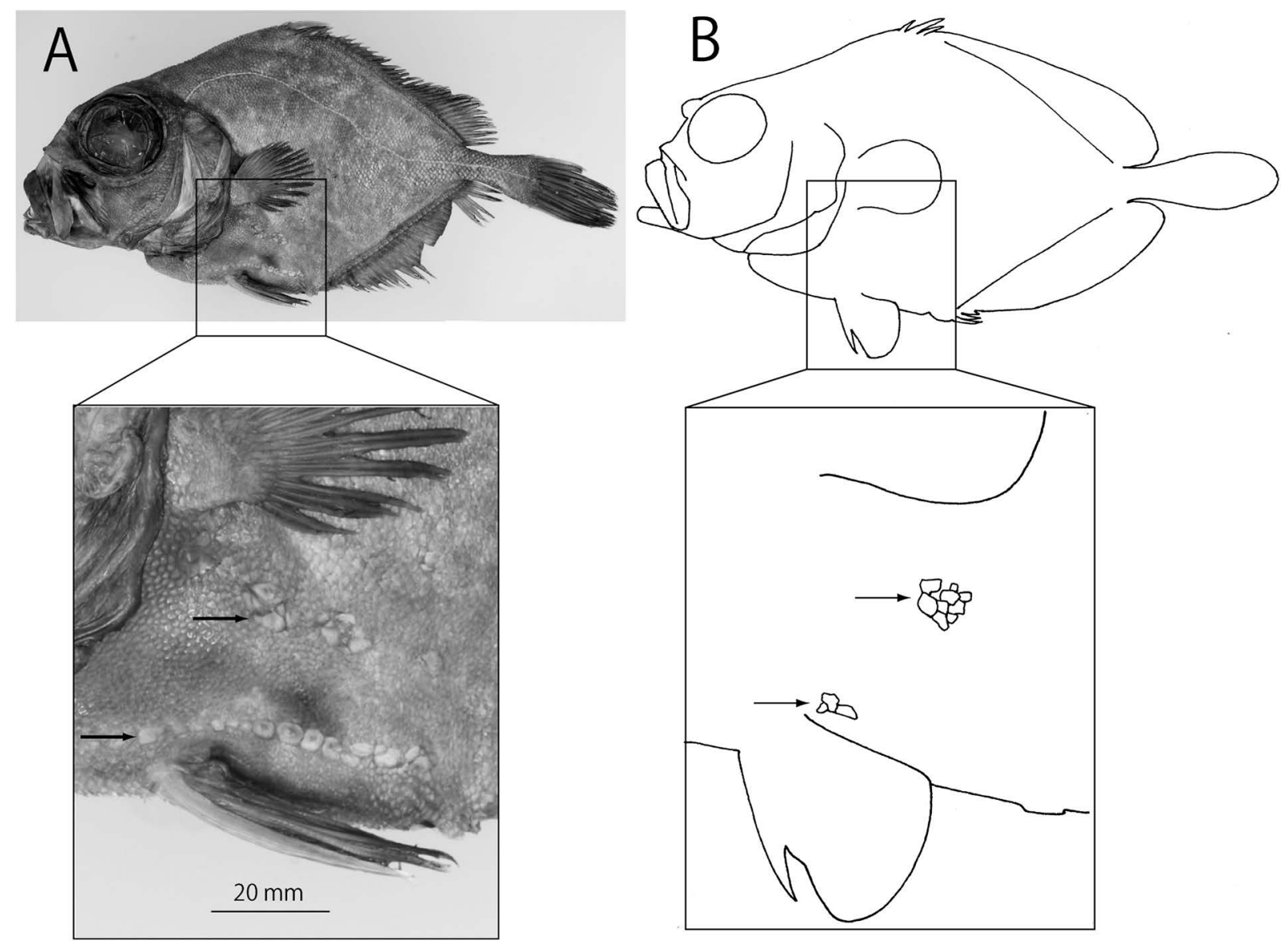

Fig. 3. Lateral aspect (above) and abdomen (below) of Allocyttus folletti. A, SNFR 10560, $293.4 \mathrm{~mm}$ SL, Emperor Seamounts; B, CAS-SU 15377, holotype of Allocyttus folletti, off California, traced from Myers (1960: fig. 1). Arrows indicate the rows of scutes.

(margin) $+1-3$ (center) (Fig. 2C), whereas in A. verrucosus it is 3-6 (margin) +1-2 (center) (Fig. 2D). James et al. (1988) described A. verrucosus as having a "row of ctenoid scales along bases of dorsal and anal fin with up to 6 spines per scale" and this agrees with our observations in A. verrucosus. These data demonstrate that the examined specimens from the Emperor Seamounts and Japan represent $A$. folletti, not $A$. verrucosus.

The two rows of abdominal scutes (serial enlarged scales), thought to be one of the diagnostic characters of $A$. verrucosus (Myers 1960; Abe and Hotta 1962; Kido 1983), were observed in the examined specimens (Fig. 3A), although their degree of development was variable. In addition, the original picture of the A. folletti holotype (Myers 1960: fig. 1) shows a small patch of somewhat enlarged scales on the mid-lateral abdomen between the pectoral and pelvic fins, and another patch above the pelvic fin (Fig. 3B). Based on their shape and position, these scale patches are akin to the scutes typical of A. verrucosus. Accordingly, the presence or absence of scutes is not a valid character to distinguish A. verrucosus from A. folletti. Mecklenburg et al. (2002) noted that $A$. verrucosus was different from $A$. folletti in having more cones (=scutes; 7 or more vs. $3-5$ in A. folletti: Mecklenburg et al. 2002; Kamiyama 2017) in the upper abdominal row. In the examined specimens, the number of scutes in $A$. folletti was $1-5$, and in A. verrucosus was 6-10 in six specimens but in one specimen (BSKU 48478), no scute was observed on either side. The variation and significance of this character needs verification.

The abdominal scutes of prejuvenile A. folletti are each a remarkably enlarged, cone-like solitary scale (e.g., Kobayashi et al. 1968; Hart 1973; Nakabo 2002), while in the examined adult specimens the scutes are an assemblage of hypertrophied scales (Fig. 3). This indicates that the state of each A. folletti scute changes ontogenetically. Mecklenburg et al. (2002) noted that the rows of scutes were reduced or lost in adults, but all the specimens examined here retained their scutes (although some were dislodged) even in the largest specimen $(537 \mathrm{~mm} \mathrm{SL})$. The degree of scute reduction in adults appears to be variable between individuals.

The characters of specimens reported as A. verrucosus from Japan and adjacent waters are presented in Table 4. They agree well with $A$. folletti but not with $A$. verrucosus (see also Tables 1, 2). Recently, Amaoka et al. (2020) published pictures of an adult and a juvenile oreosomatid (supposedly from Hokkaido, Japan) identified as A. folletti. In the adult specimen, the mouth is strongly projected, negating the value of any measurements from the snout tip (e.g., SL1) and thus it is not listed in Table 4. However, from the measurement of the picture of the adult specimen, the percentage in SL2 of BD1 (53.0\%), BD2 (53.5\%) and CPL2 (11.0\%) agree with $A$. folletti but not A. verrucosus (see 


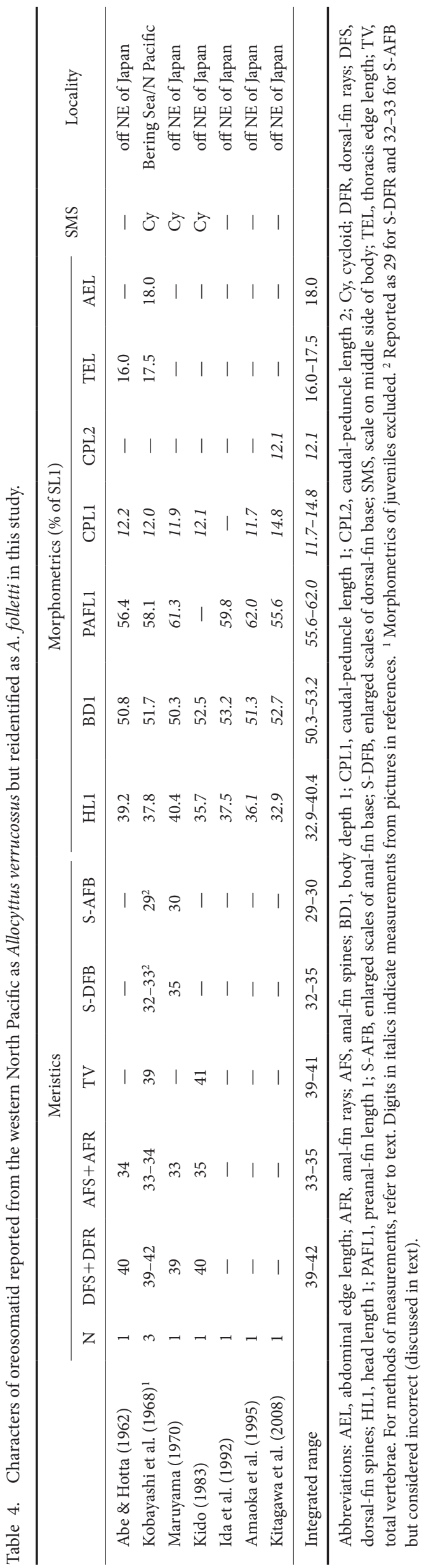

Table 3). HL2 is $39.2 \%$ of SL2 (beyond the range of A. folletti) but this variation is probably due to damage of the opercular elements, viz., it appears the subopercle and interopercle are lost and the opercle is rotated backward from its original position. We therefore consider these individuals to represent $A$. folletti, not $A$. verrucosus.

We conclude that the oreosomatid of the western North Pacific Ocean is A. folletti, supporting the arguments of James et al. (1988), Mecklenburg et al. (2002) and Nakabo and Kai (2013).

Distinction of $A$. folletti from $A$. verrucosus. Characters seemingly useful for distinguishing $A$. folletti and A. verrucosus in Tables 1-3 are compared with literature data (Table 5) to test their validity. In the meristic characters, dorsal-fin spines+rays (36-42 vs. 33-38), anal-fin spines + rays (31-35 vs. $27-33$ ), and total vertebrae (37-41 vs. 34-38) are useful, although the ranges of the two species overlap. S-DFB (31-42 vs. 26-31) and S-AFB (29-37 vs. 2528) are likely to be useful, although the available data from references are limited. Kobayashi et al. (1968) reported that based on three specimens the number of "rough scales along to dorsal base" (=S-DFB) was 29 , and the number of "rough scales along to anal base" (=S-AFB) as $32-33$. However, the correct counts of S-DFB and S-AFB are likely to be 32-33 and 29 respectively, because the number of S-DFB is greater than the number of S-AFB in most (21 of 23) of the specimens examined here; it is not likely that S-DFB was fewer than S-AFB in all of the three specimens of Kobayashi et al. (1968). Unfortunately, the picture of the specimen in Kobayashi et al. (1968: pl. 1a) is not clear enough to count the number of S-DFB and S-AFB correctly, although the rough counts based on an enlarged copy (200\%) of the picture were ca. 33 and ca. 30 respectively. Ward et al. (1996) raised the number of pectoral-fin rays as a character to distinguish A. folletti and A. verrucosus (19-21 vs. 17-19 respectively). However, the data of the present study (Table 1) and those of the references in Table 5 revealed the wider overlapping range (19-21 vs. 17-21 respectively).

In the morphometric characters as \% of SL1 (Table 5; data of juveniles are neglected), shorter PAFL1 (53.8-63.6\% vs. 64.8-83.7\%) and longer CPL2 (10.4-15.6\% vs. $6.1-10.2 \%)$ seem especially useful because the two species were clearly separated by these characters. HL1 (32.9-40.4\% vs. 38.548.4\%), TEL (14.6-18.5\% vs. $17.8-28.0 \%)$, AEL (14.6-20.2\% vs. $20.1-26.0 \%)$ and CPL1 (11.2-14.8\% vs. $7.7-11.8 \%)$ seem useful although the ranges of the two species overlap. The range of $\mathrm{BD} 1$ in $A$. verrucosus (46.0-77.9\%) includes the whole range in A. folletti (50.3-55.9\%). However, the data of A. verrucosus in James et al. (1988) from the specimens of South Africa (46.0-73.3\%) and Australia (53.5-77.9\%) include exceptionally low values ( $46.0 \%$ and $53.3 \%$ ), which might be mistakes or abnormalities. If these values are excluded, available data of this character in A. verrucosus is $56.5-77.9 \%$, clearly separable from $A$. folletti (50.3-55.9\%). The significance of the last character needs further verification.

The caudal peduncle in Allocyttus folletti is deeper posteriorly compared to that of $A$. verrucosus (Fig. 1C, D); when the depth of caudal-fin base (CFD) is expressed as a per- 


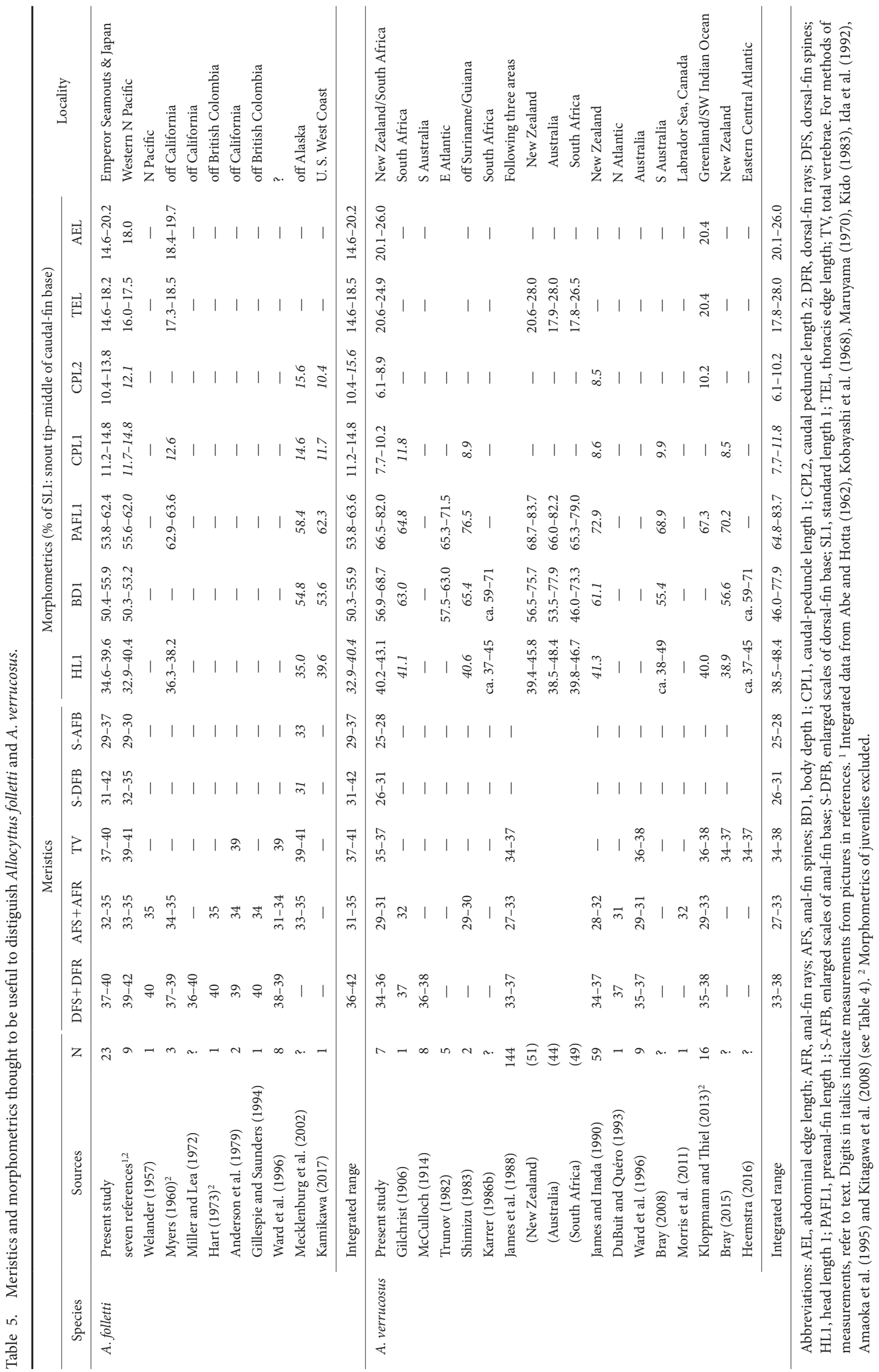


centage of caudal-peduncle depth (140-188\% vs. $111-127 \%$ in the examined specimens) it seems a useful character. This can be seen in the figures in the references (cited in Tables 4, 5) although accurate measurement based on these figures is difficult in many cases.

Ward et al. (1996) pointed out that A. folletti has a narrower lachrymal width (LW) than in A. verrucosus (1.7$2.4 \%$ vs. $3.3-4.0 \%$ of SL respectively). In our observation, this character clearly separated the two species (Tables 2, 3), supporting the validity of this character. But note that SL of Ward et al. (1996) was measured from between lachrymal tips anteriorly, and thus cannot be directly compared with our study. Ward et al. (1996) pointed out that A. folletti has longer fin spines (e.g., 2nd dorsal-fin spine: $7.6-12.6 \%$ vs. $5.4-9.1 \%$ of SL), but from the wide range of overlapping, it is often difficult to identify the specimens by this character alone (see also Tables 2, 3).

Scales of mid-side (SMS) are cycloid in A. folletti and ctenoid in A. verrucosus (Fig. 2A, B) making this a useful character to separate the two species. Furthermore, the numbers of spines on the margin of S-DFB and S-AFB (up to 7-12 vs. up to 3-6) differ between the two species based on the examined specimens. The data in the holotype of $A$. folletti (up to nine) and A. verrucosus in James et al. (1988) (up to six) agree with our observations.

The adherence of the scales was thought to be a character to separate the two species. Abe and Hotta (1962) identified their specimen of oreosomatid from Japan as A. verrucosus and not $A$. folletti partly because of the "tenacious" (= adherent) scales, in addition to the scutes. Direct comparisons of the two species revealed that the mid-side scales are, overall, preserved better in A. verrucosus, and those scales were more easily removed by tweezers in A. folletti. However, Abe and Hotta (1962) apparently judged the scale condition as "tenacious" without comparison with the "real" A. verrucosus. In the specimen of Abe and Hotta (1962: figs $1,3)$ the scales look well preserved; this may have led to these authors describing the scales as "tenacious" and misidentifying the specimen as $A$. verrucosus.

Myers (1960: 93) listed the following "subtle features" to distinguish $A$. folletti from $A$. verrucosus: (1) more concave predorsal profile, (2) flatter belly, (3) snout less smoothly rounded down, and (4) more prominent and rugose nasal boss (=bony projection). Regarding the predorsal profile (1), however, in A. folletti it was usually (in 18 of 23 specimens examined) slightly convex between the dorsal-fin origin and the vertical through the posterior end of the gill opening (Figs 1A, 3). In A. verrucosus, the corresponding part was slightly concave (Fig. 1B) in six of seven comparative specimens. The same conditions can be observed in various photos and illustrations of $A$. folletti [e.g., Myers 1960 (holotype: Fig. 3); Abe and Hotta 1962; Kobayashi et al. 1968; Machida 1984; Mecklenburg et al. 2002; Amaoka et al. 2020), and those of A. verrucosus (e.g., Gilchrist 1906; McCulloch 1914; Shimizu 1983; James et al. 1988; James and Inada 1990; Bray 2008, 2015; Kloppmann and Thiel 2013). However, the relevant part of the predorsal contour was straight in some specimens of both species, and thus this character is not always useful to distinguish the two species. Regarding the shapes of the belly (2) and snout (3), we did not recognize any significant differences between the two species. The nasal boss (4) was more strongly projected (Fig. $1 \mathrm{E}, \mathrm{F}$ ) and armed with larger spinules in A. folletti (except the smallest specimen examined: NSMT-P 30689, $190.7 \mathrm{~mm}$ SL), as described by Myers (1960).

Note on distribution. In the Emperor Seamounts, Allocyttus folletti was previously reported from Koko Seamount $\left(35^{\circ} 30^{\prime} \mathrm{N}, 171^{\circ} 30^{\prime} \mathrm{E}\right)$ and northward to Suiko Seamount $\left(44^{\circ} 34^{\prime} \mathrm{N}, 170^{\circ} 24^{\prime} \mathrm{E}\right)$ (see Mundy 2005). The present study extends the range southward from Koko Seamount to Hancock Seamounts. The latter comprises two seamounts, Southeast Hancock $\left(29^{\circ} 48^{\prime} \mathrm{N}, 178^{\circ} 05^{\prime} \mathrm{E}\right)$ and Northwest Hancock $\left(30^{\circ} 17^{\prime} \mathrm{N}, 178^{\circ} 44^{\prime} \mathrm{E}\right)$ (Boehlert 1988). The specimen we examined (FAKU 119906) was collected from Hancock Seamounts but its exact locality is unknown.

Biological features and management suggestions. The largest specimen examined in the present study was $537 \mathrm{~mm}$ SL $(630 \mathrm{~mm}$ in total length $=\mathrm{TL})$ considerably larger than the largest specimen of A. verrucosus in the review of James et al. (1988), $265 \mathrm{~mm}$ SL $(320 \mathrm{~mm} \mathrm{TL})$. The maximum size of A. verrucosus reported by Lyle and Smith (1997) in an abundance and biological survey of oreos in south-eastern Australia was ca. $390 \mathrm{~mm}$ TL (the corresponding SL is unknown but estimated as ca. $325 \mathrm{~mm}$ ). These data suggest that $A$. folletti reaches a larger maximum size than $A$. verrucosus.

Stewart et al. (1995) estimated that for A. verrucosus from western Bass Strait, Australia, the age at maturity was 28 years in females and 24 years in males, and the maximum age was $130-170$ years for fish of $34-35 \mathrm{~cm}$ TL. From the estimated low fecundity, high age at maturity and longevity, Stewart et al. (1995) noted the susceptibility of A. verrucosus to over-exploitation. Although the age, growth, and maturity of $A$. folletti are not understood, similar biological features are anticipated due to its close affinity with, and similar deep-sea habitat to, A. verrucosus. Collecting biological information of this species, including its life history and genetics, under the valid scientific name $A$. folletti, is required to understand its subpopulations and vulnerability, and to sustainably manage its exploitation.

\section{Acknowledgements}

We are grateful to Dr. Gento Shinohara (NSTM-P), Drs. Yoshiaki Kai, Naohide Nakayama and Takashi Sato (FAKU), and Dr. Hiromitsu Endo (BSKU) for loaning specimens. Dr. Koichi Shibukawa (Museum of Natural and Environmental History, Shizuoka) provided helpful advice. Mr. G. K. Yearsley kindly corrected an earlier English draft and provided helpful advice. Ms. Yuko Matsunaga (SNFR) assisted in preparation and registration of the specimens. We appreciate the effort of the officers and crew of F/Vs Tomi-maru, Seishin-maru No. 88 and Kaiyo-maru No. 38 for collecting specimens. This study was funded through the "Research and assessment program for internationally managed fisheries resources, the Fishery Agency of Japan, through which 
the examined specimens registered in SNFR were captured, including those by F/V Shoshin-maru No. 88 in the Program of Scientific Observer of NPFC (The North Pacific Fisheries Commission). We are thankful to Drs. Takeshi Hayashibara, Takashi Yanagimoto, Shiro Yonezaki, Takehiro Okuda, Kazuya Nishida and Mai Miyamoto, Mitsuaki Kitamura and Chisato Murakami, who joined the promotion of the project.

\section{References}

Abe, T. and Hotta, H. 1962. An addition to the Japanese Piscifauna. Japanese Journal of Ichthyology 8: 152-156.

Amaoka, K., Nakaya, K., and Yabe, M. 1995. The Fishes of Northern Japan. Kita-Nihon Kaiyo Center, Sapporo, 390 pp. [In Japanese]

Amaoka, K., Nakaya, K., and Yabe, M. 2011. Fishes of Hokkaido. The Hokkaido Shimbun Press, Sapporo, 482 pp. [In Japanese]

Amaoka, K., Nakaya, K., and Yabe, M. 2020. Pictorial Guide to the Fishes of Hokkaido. The Hokkaido Shimbun Press, Sapporo, 590 pp. [In Japanese]

Anderson, M. E., Cailliet, G. M., and Antrim, B. S. 1979. Notes on some uncommon deep-sea fishes from the Monterey Bay area, California. California Fish and Game 65: 256-264.

Boehlert, G. W. 1988. Current-topography interactions at mid-ocean seamounts and the impact on pelagic ecosystem. GeoJournal 16: 45-52.

Borets, L. A. 1986. Ichthyofauna of the Northwestern and Hawaiian Submarine Ranges. Journal of Ichthyology 26(3): 1-13.

Bray, D. J. 2008. Family Oreosomatidae. Pp. 441-446. In: Gomon, M., Bray, D., and Kuiter, R. (Eds) Fishes of Australia's Southern Coast. Reed New Holland, Sydney.

Bray, D. J. 2015. 147 Family Oreosomatidae. Pp. 1041-1048. In: Roberts, C. D., Stewart, A. L., and Struthers, C. D. (Eds) The Fishes of New Zealand. Volume Three. Te Papa Press, Wellington.

Cook, S. F. and Long, J. 1985. The oxeye oreo, Allocyttus folletti Myers, from the Bering Sea. California Fish and Game 71: 57.

DuBuit, M.-H. and Quéro, J.-C. 1993. Premier signalement et Atlantique nord-est, d'Hoplostethus cadenati (Beryciformes, Trachichthyidae) et d'Allocyttus verrucosus (Zeiformes, Oreosomatidae). Cybium 17: 81-82.

Eschmeyer, W. N. and Herald, E. S. 1983. A Field Guide to Pacific Coast Fishes of North America. Houghton Mifflin Company, Boston, xiv +336 pp., 48 pls.

Fricke, R. and Eschmeyer, W. N. 2019. A guide to fish collections in Eschmeyer's Catalog of Fishes. Available at http://researcharchive. calacademy.org/research/ichthyology/catalog/collections.asp (21 May 2019).

Gilchrist, J. D. F. 1906. Description of fifteen new South African fishes, with notes on other species. Marine Investigations in South Africa 4: 143-171, pls 37-51.

Gillespie, G. E. and Saunders, M. W. 1994. First verified record of the shortfin mako shark, Isurus oxyrhinchus, and second records or range extensions for three additional species, from British Columbia waters. The Canadian Field-Naturalist 108: 347-350.

Grande, T. C., Borden, W. C., Wilson, M. V. H., and Scarpitta, L. 2018. Phylogenetic relationships among fishes in the order Zeiformes based on molecular and morphological data. Copeia 106: 20-48.

Hart, J. L. 1973. Pacific Fishes of Canada. Bulletin 180. Fisheries Research Board of Canada, Ottawa, ix +740 pp.

Heemstra, P. C. 2016. Oreosomatidae. Pp. 2206-2215. In: Carpenter, K. E. and Angelis, N. D. (Eds) FAO Species Identification Guide for Fishery Purposes. The Living Marine Resources of the Eastern
Central Atlantic. Vol. 3. Bony Fishes Part 1 (Elopiformes to Scorpaeniformes). Food and Agriculture Organization of the United Nations, Rome.

Hughes, S. E. 1981. Initial U. S. exploration of nine Gulf of Alaska seamounts and their associated fish and shellfish resources. Marine Fisheries Review 43: 26-33.

Ida, H., Asahida, T., Saito, Y., and Terashima, H. 1992. Fishes of Iwate. Iwate Nippo Co., Ltd., Morioka, 216 pp. [In Japanese]

Inada, T. 1997. Oreosomatidae. P. 168. In: Okamura, O. and Amaoka, K. (Eds) Sea Fishes of Japan. Yama-Kei Publishers Co., Ltd., Tokyo. [In Japanese]

James, G. D. and Inada, T. 1990. Allocyttus verrucosus (Gilchrist, 1906). P. 221. In: Amaoka, K., Matsuura, K., Inada, T., Takeda, M., Hatanaka, H., and Okada, K. (Eds) Fishes Collected by the R/V Shinkai Maru around New Zealand. Japan Marine Fishery Resource Research Center, Tokyo.

James, G. D., Inada, T., and Nakamura, I. 1988. Revision of the oreosomatid fishes (family Oreosomatidae) from the southern oceans, with a description of a new species. New Zealand Journal of Zoology 15: 291-326.

Kamikawa, D. J. 2017. Survey Fishes: an illustrated list of the fishes captured during the Northwest Fisheries Science Center's fishery resource analysis and Monitoring Division's West Coast surveys. NOAA Technical Memorandum NMFS-NWFSC (138): i-xv+1437.

Karrer, C. 1986a. Oreosomatidae. Pp. 775-776. In: Whitehead, P. J. P., Bauchot, M.-L., Hureau, J.-C., Nielsen, J., and Tortonese, E. (Eds) Fishes of the North-eastern Atlantic and the Mediterranean. Volume II. UNESCO, Paris.

Karrer, C. 1986b. Family No. 139: Oreosomatidae. Pp. 438-440. In: Smith, M. M. and Heemstra, P. C. (Eds) Smiths' Sea Fishes. Springer-Verlag, Berlin.

Kido, K. 1983. Allocyttus verrucosus (Gilchrist). Pp. 126-127, 203. In: Amaoka, K., Nakaya, K., Araya, H., and Yasui, T. (Eds) Fishes from the North-Eastern Sea of Japan and the Okhotsk Sea off Hokkaido. The Intensive Research of Unexploited Fishery Resources on Continental Slopes. Japan Fisheries Resource Conservation Association, Tokyo.

Kitagawa, D., Imamura, H., Goto, T., Ishito, Y., Fujiwara, K., and Ueda, Y. 2008. Field Guide to the Fishes from the Tohoku District, Northeastern Waters of Japan. Tokai University Press, Tokyo, xvii +140 pp.

Kloppmann, M. H. F. and Thiel, R. 2013. First record of the warty oreo, Allocyttus verrucosus (Gilchrist, 1906), in Greenland waters. Journal of Applied Ichthyology 29: 451-455.

Kobayashi, K., Mikawa, M., and Ito, J. 1968. Descriptions of the young and one immature adult specimens of coster dory, Allocyttus verrucosus (Gilchrist) from the northern part of the Pacific. Bulletin of the Faculty of Fisheries Hokkaido University 19: 1-5.

Lyle, J. M. and Smith, D. C. 1997. Abundance and biology of warty oreo (Allocyttus verrucosus) and spiky oreo (Neocyttus rhomboidalis) (Oreosomatidae) off south-eastern Australia. Marine Freshwater Research 48: 91-102.

Machida, Y. 1984. Family Zeidae. P. 118, pl. 103. In: Masuda, H., Amaoka, K., Araga, C., Uyeno, T., and Yoshino, T. (Eds) The Fishes of the Japanese Archipelago. Tokai University Press, Tokyo.

Maeda, K. and Tsutsui, D. 2003. List of fishes of Hokkaido. Pp. 481-504. In: Ueda, Y., Maeda, K., Shimada, H., and Takami, T. (Eds) Fisheries and Aquatic Life in Hokkaido. The Hokkaido Shimbun Press, Sapporo. [In Japanese]

Maruyama, K. 1970. Some deep-water fishes from off the Tohoku and adjacent regions. Bulletin of Tohoku Regional Fisheries Research Laboratory (30): 43-66. [In Japanese]

Maruyama, K. 1971. A catalogue of fishes of Iwate Prefecture, Japan. 
Bulletin of Iwate Prefectural Fisheries Experimental Station (1): 1-70. [In Japanese]

McCulloch, A. R. 1914. Report on some fishes obtained by the F. I. S. "Endeavour" on the coasts of Queensland, New South Wales, Victoria, Tasmania, South and South-western Australia. Part II. Biological Results of the Fishing Experiments Carried on by the F. I. S. "Endeavour," 1909-14 2: 77-165, pls 13-34.

Mecklenburg, C. W., Mecklenburg, T. A., and Thorsteinson, L. K. 2002. Fishes of Alaska. American Fisheries Society, Bethesda, MA, U. S. A., xxxvii+1037 pp.

Miller, D. J. and Lea, R. N. 1972. Guide to the coastal marine fishes of California. California Fish Bulletin (157): 1-249.

Morris, C. J., Coad, B. W., and Fisher, G. 2011. The warty dory, Allocyttus verrucosus new to the fish fauna of Canada. The Canadian Field-Naturalist 125: 74-75.

Mundy, B. C. 2005. Checklist of the fishes of the Hawaiian Archipelago. Bishop Museum Bulletin in Zoology (6): 1-704.

Myers, G. S. 1960. A new zeomorph fish of the family Oreosomatidae from the coast of California, with notes on the family. Stanford Ichthyological Bulletin (4): 89-98.

Nagtegaal, D. A. 1983. First record of an adult oreo, Allocyttus folletti Myers, from British Columbian waters. Syesis 16: 89-90.

Nakabo, T. 1993. Oreosomatidae. Pp. 467, 1290. In: Nakabo, T. (Ed.) Fishes of Japan with Pictorial Keys to the Species. Tokai University Press, Tokyo. [In Japanese]

Nakabo, T. 2000. Oreosomatidae. Pp. 508, 1505-1506. In: Nakabo, T. (Ed.) Fishes of Japan with Pictorial Keys to the Species. Second Edition. Tokai University Press, Tokyo. [In Japanese]

Nakabo, T. 2002. Oreosomatidae. Pp. 508, 1502. In: Nakabo, T. (Ed.) Fishes of Japan with Pictorial Keys to the Species. English Edition. Tokai University Press, Tokyo.

Nakabo, T. and Kai, Y. 2013. Oreosomatidae. Pp. 601, 1902. In: Nakabo, T. (Ed.) Fishes of Japan with Pictorial Keys to the Species. Third Edition. Tokai University Press, Hadano. [In Japanese]

Nishida, K., Murakami, C., Yonezaki, S., Miyamoto, M., Okuda, T., and Kiyota, M. 2016. Prey use by three deep-sea fishes in the Emperor Seamount waters, North Pacific Ocean, as revealed by stomach contents and stable isotope analyses. Environmental Biology of Fishes 99: 335-349.

Sheiko, B. A. and Fedorov, V. V. 2000. Chapter 1. Class Cephalaspido-
morphi-Lampreys. Class Chondrichthyes-Cartilaginous Fishes. Class Holocephali-Chimaeras. Class Osteichthyes-Bony Fishes. Pp. 7-69. In: Moiseev, R. S. and Tokranov, A. M. (Eds) Catalog of Vertebrates of Kamchatka and Adjacent Waters. Kamchatskiy Petchatniy Dvor, Petropavlovsk-Kamchatsky. [In Russian]

Shimizu, T. 1983. Oreosomatidae. Pp. 288-289. In: Uyeno, T., Matsuura, K., and Fujii, E. (Eds) Fishes Trawled off Suriname and French Guiana. Japan Marine Fishery Resource Research Center, Tokyo.

Stewart, B. D., Fenton, G. E., Smith, D. C., and Short, S. A. 1995. Validation of otolith-increment age estimates for a deepwater fish species, the warty oreo Allocyttus verrucosus, by radiometric analysis. Marine Biology 123: 29-38.

Tokranov, A. M., Orlov, A. M., and Biryukov, I. A. 2004. Distribution and size-weight composition of some rare fish species in Pacific waters off the northern Kurile Islands and southeast Kamchatka. Journal of Ichthyology 44: 208-216.

Trunov, I. A. 1982. Zeiformes of the thalassobathyal of the southeastern Atlantic. Byulleten Moskovskogo Obshchestva Ispytatelei Prirody Otdel Biologicheskii 87: 41-53. [In Russian]

Tyler, J. C. and Santini, F. 2005. A phylogeny of the fossil and extant zeiform-like fishes, upper Cretaceous to recent, with comments on the putative zeomorph clade (Acanthomorpha). Zoological Scripta $34: 157-175$.

Tyler, J. C., O'Toole, B., and Winterbottom, R. 2003. Phylogeny of the genera and families of zeiform fishes, with comments on their relationships with tetraodontiforms and caproids. Smithsonian Contributions to Zoology (618): i-iv+1-110.

Ward, R. D., Elliott, N. G., Yearsley, G. K., and Last, P. R. 1996. Species and Stock Delineation in Australasian Oreos (Oreosomatidae). Final Report to Fisheries Research and Development Corporation. Grant number 92/94. CSIRO Division of Fisheries, Hobert, $\mathrm{ii}+144+1.1-3.5 \mathrm{pp}$.

Welander, A. D., Johnson, R. C., and Hajny, R. A. 1957. Occurrence of the boar fish, Pseudopentaceros richardsoni, and the zeid Allocyttus verrucosus, in the north Pacific. Copeia 1957: 244-246.

Yearsley, G. K. and Last, P. R. 1998. Neocyttus psilorhynchus, a new oreosomatid (Pisces, Zeiformes) from southern Australia and New Zealand, with redescriptions of its congeners. New Zealand Journal of Marine and Freshwater Research 32: 555-579. 